\title{
THE IMPACT OF VIOLENT MOVIES
}

\author{
Muhammad Uzair Khan \\ University of Karachi
}

\begin{abstract}
This research work includes a study of the effects of violent films on teenagers and children. In this research, it has been identified that what is the impact of violence on teenagers physically and psychologically. Films, whether Bollywood, Hollywood, or Pakistani Cinema, promote a brutal culture in society with their movies. Children and teenagers are becoming aggressive every day by watching these violent movies. The research article has raised the issue of the spread of ferocity in humanity through these films. The study has used several articles to explore the research.
\end{abstract}




\section{INTRODUCTION}

\section{Research Backdrop}

The importance of the media in the contemporary world is gigantic. In human history, the media has had such a significant impact on our lives, attitudes, and practices. Despite this, the media plays an extraordinary and essential role in promoting social presentations, perspectives, and global beliefs. The media refers to understanding how it shapes our way of life. Movies and television programs have mainly influenced our society. Moreover, movies have always been considered as the most popular and engaging media.

The most significant change in our society is to be immersed in our way of life and done by the media. In this new society, radio, television, film, video, games, mobile phones, and computers are assumed to occupy a prominent position in our society. The media is getting scarcer and has dramatically affected our beliefs, quality, and practice. Movies can be described as the signals of electronics for moving images, pictures, and electronic signs of images that are associated with anomalous and sturdy sounds, the content of which is used for training, education entertainment, and the like. The best multimedia tool is the movie. This is an extremely gentle way of conveying information to the masses. This can be an excellent way to match and clarify and get their information.

The film plays a significant role in the formation and influence of the world. An individual who is greatly influenced by movies tries to build an opinion that can significantly affect society. Movies are vital to everyone's life. It is hard to imagine existence without them. From children to teenagers and adults to maturity, everyone sees movies effectively and weirdly. Today, there is an enormous trend in movies with negative and positive angles. It is difficult to judge whether the impact of movies influences positively or negatively, but it depends entirely on how it is obtained. In this regard, the media also play a significant role in highlighting the issues of the people. So now, the media has become a source of data, deviations, and learning with nervousness, discouragement, violence, and hostility.

\section{Statement of the problem}

To observe the effect on the behavior of teenagers by violence shown in movies and to critically inspect their behavior towards such movies.

\section{The rationale of the study}

The vital reason for this research is to focus on studying the effects of violence that appeared in the films about teenager's behavior when they leave the scene in which these elements are more and more attractive and their predominant center of interest. With the advent of the media, which include movies, television, computers, and especially the evolution in media known as the Internet, children and teenagers are receiving more and more aggressive pictures around the globe. In many countries, even though we believe that the main factors of violent behavior of young people are the situation of their families and the financial conditions in which they survive to find themselves. 
In Pakistan, the majority of viewers watch Indian movies. For the past five years, the increase of Hollywood movies has been seen, and lastly, the Pakistan film industry. The curse of aggressiveness and violence increases in our society, and primarily it targets the mind of teenagers. The study has designed to find the significant influence of these movies on Pakistani teenagers. The study has included both male and female participants.

\section{Research Objectives}

The main objectives of this research are:

To observe how teenagers are being affected by violence shown in movies?

To examine and understand the significant part of movies as a fascination for violence in the lives of teenagers.

The study has conducted to analyze the level of violence in all types of movies.

To observe the mean and type of mass media use between teenagers.

To identify the most vital and common feature use in vicious movies.

To analyze the relation between aggressive behaviors of teenagers and media violence.

\section{Significance of the study}

Research is needed for people affected by aggressive content. This encourages them to reflect on the influence and causes of these films. This is useful for people who see why these violent films gradually affect teenagers. How does this affect their lives, and how are they attracted to them? This is fundamental because the media are the dominant power and provide data and stimulate the entire population. Some people say that violent films are just entertainment and are not interested at all. The notion of severity for teenagers. Thus, population assumptions about violence are formed simply by the mass media. People regularly see violence, and this is common in our society. Similarly, our young people watch this and accept dynamic and wild behavior, as is appropriate in our society.

\section{LITERATURE REVIEW}

The reason for this chapter is to check the literature of various authors on the relevant topic. For example, in search, selection, and solutions to test questions, it is recommended to use different data sources; books, magazines, newspapers, and websites. The focus of this review is to determine whether a film is genuinely aggressive and can create violence among adolescents. Researchers' research on the choice of violence as the subject of the exam seems evident because it is inevitable in teen films (Pavlova \& Silbereisen, 2015). After all, it creates power. The study can be guided by previous studys. Also, experience helps to prove the feasibility of the investigation. Since 1995, a large number of comments, reports, and articles on violent films have been published for a long time (Huesmann, 2007). The ongoing research in media effect research is whether film violence can lead to broader issues of hatred and violence among young people. Some researchers believe that the cause of violence is harmful, while others do not. 


\section{Society and Violence}

Violence is not something new in the society for years. In the contemporary world, it's hard to escape from its similarities with projects, movies, recordings, children's programs, and even commercials (Barlińska et al., 2013). Counterfeiting, hostility, etc. are important fears of all societies. In the past, many researchers have focused on this situation and results. This problem has been intense with time and innovation. In the United States or other Western countries, researchers benefit from the incredible reflection of this issue. However, for countries like Pakistan, their researchers did not respond. This review reveals the impact of cruel movies on Pakistani youth. The results of this review show that many people report using this type of film as a model for any crime.

Many researchers believe that the proximity of firearms in a movie can lead to hostility. This study analyzes the springs used by weapons analyzed in the best movies. Their results show that the violence in the movie has multiplied by 19 years until now. These results indicate that if our children do not use these firearms after watching these movies, they also ask the producers for giving them a reason and content (Dai et al., 2015).

Violence in the media has increased driving when drunk. The study abused different kinds of violence in very successful films to determine whether barbaric behavior would magnify atrocities. They make it easy to confirm life. In general, they found that violence in the media would affect people in the short term, and their configuration did not allow them to investigate long-term consequences.

\section{Media and Its Influence on People}

There is no end to this kind of discussion, when does the media have an impact on people, and how do you examine these effects? This research verifies that images are not limited to television shows, movies, videos, games, and network effects on people with thoughts, emotions, and feelings for a short time, which increases the power and impact of horror, especially among young people (Anderson $\&$ Warburton, 2012). These confirmations can create conflicts when affecting adolescents and also have long-term effects.

Disclosure of violence on television, movies, mobile phones, games, and the Internet may create behavioral problems. More introductions to rough electronic media can be dangerous for children and children, and children continue to use force. Young people act in the short term, but children's behavior is long-term. Not only is this dangerous driving, but it also creates the same number of different factors, such as threats to public health (Cardwell, 2013).

The study has determined how young people saw violent movies in scandals. The study shows that they are getting more and more ferocious and cruel. Movie violence has never been included in television violence because it is a valuable donor whose barbarism is more inconsistent than violence on television. 


\section{Role of Media to create a Perception}

Electronic media have an incredible version of sense that can create the world for us. It was pointed out that Egypt, the pyramids, and the pictures of the mummies surrounded the bell, even though we never went to Egypt (Wang et al., 2012). People who watch barbaric and influential films have a keen sense of violence. Because it depends on their assessment of the violence and its perceptions.

"For things like 20 years, most psychology research networks are effectively combined with media research, that is, television violence has increased dominant behavior, discomfort, and mental health effects (Cardwell, 2013). Young people found that most violations are usually represented graphically. Why did all this violence happen? Since groups such as violence and film have been well received in the film industry, more and more people are participating. Producers are not worried about the possibility of desensitization and dehumanization of the general population on Earth. They only need their books in the organization. The number of projectiles in a raw film can complete the entire group.

The investigation into the increasingly vicious content in movies and videos concerns children and teenagers guilty of wrongdoing. The study shows that people who observed barbaric behavior during their youth were powerful in adulthood. When they saw the violent children on the screen, they began to think about it, which led to hatred, desensitization of feelings of sympathy, and the use of violence (Ali \& Ali, 2015).

Music and movie stations have a great passion for our childhood. The glamorous films from the outside make them happy with their crazy personality. They are so fascinated and even try to have a brilliant lifestyle. This cultural attack affected our childhood and neglected our social goodness and religious qualities. The explanation of the violence we aroused in our society in our childhood dates back to the irrational impact of these projects.

\section{Not Easy to Escape from Media}

Many people invest their energy in front of television and movies, using the Internet, mobile phones, etc. Especially teenagers, who suffer more from movies and videos. They get what they see on the screen more effectively. Also, thanks to the development of advanced technologies, they download movies from the Internet. Because of this option of downloading, teens lose a lot of time watching movies (Wartella et al., 2016). They are not developed ample to choose what they are looking for, whether it is good or not, and thus they observe a wide variety of things.

For instance, turn on the television, the violence is there or in a film, the latter exists. The reality can be overcome: if the individual begins to live in a world of anecdotes, then the world of anecdotes has become their existence and reality. The effect of the description of violence, which is related to the collection of practices of recognition and learning of individuals shows in movies (Matrix, 2014). Currently, there is a major trend in the movies to show violence because of its TRP. Each film has a brutal behavior that hurts young people (Jang et al., 2015). Teenagers are not able 
to separate violence in recreation and violence in real life. Our moral obligation is, therefore, to protect teenagers from exposure to inappropriate violence. Sadly, Filmmakers promote violence in their movies to provoke the young mind of teenagers.

\section{METHODOLOGY}

The method of study used to research in natural and social sciences is called methodology. The research can be conducted using quantitative or qualitative methods, according to the significance of the study. This research article has been undertaken with the help of a secondary qualitative approach, in which various articles used to analyze the impact of violent movies on teenagers, focusing on Pakistan.

\section{DISCUSSION}

As a critical mass media, the movie impacts the audience's movie-watching experience in a vivid interpretation. In this era of entertaining pan-entertainment, what adverse effects will movie culture have on teenagers under the prevalent background of entertainment? In the previous influence, it was mentioned that film culture has a positive influence on the socialization process of teenagers. Still, this influence is to use the double-edged sword as a social group with its characteristics. Teenagers are age, knowledge, and experience. Other aspects show different characteristics than adults. In the film, the promotion of non-mainstream culture, to cater to the meaning of commercial effects, produce content that is contrary to the values of the audience, especially teenagers, that is, the melting of traditional moral concepts and distorting the positive and correct value orientation.

The mass media is a powerful tool that can serve both good and evil, and generally speaking, if it is not adequately controlled, it may be malignant. "The main way of learning for teenagers is observation and imitation. Faced with the violent plot in the movie, they cannot distinguish the correctness of things, so it is easy to imitate after watching the movie, and some will appear in real life.

Stereotypes are generalized or hypothetical conceptualizations of a group of individuals, which will lead to the homogenization of the individuals in the group. That is, most people in the group are perceived as having the same characteristics. Stereotypes can also create a biased expectation of how to think, feel, and behave about other people's surface characteristics. For teenagers, the media, including movies, are often full of gender and ethnic stereotypes. And they can be exposed to some negative information in the movie, such as drugs, alcoholism and other scenes. The rendering of the movie plot will affect the teenagers' understanding of these behaviors. It may develop from the original attempt into a stable material user until material dependence is formed, which is worthy of our consideration. 
Teenagers' thoughts are still in the process of growth, and values have not yet been fully formed. When faced with a lot of complex information, they are very vulnerable to some evil thoughts. The creators of the film should pay more attention to the group of teenagers and create film and television works suitable for them. They should bear the social attributes and responsibilities of entertaining and help teenagers build positive values and health psychology. Bandura proposed in his social learning theory that moral behavior can be obtained through learning and can be changed. The observer imitates and learns the observed objects, reveals the process of learning models in a linear order, and observes and directly learns. It is necessary to establish a role model for peers and a role model for adults in movies.

\section{CONCLUSION}

The movie should not only show incredible visual effects but also pay attention to the function of educational guidance, especially the Pakistani cinema industry. The film is an art, and the artistic appeal of the film should be used to attract teenagers, not straightforward education. In a period of relatively weak views of right and wrong, creators should do an excellent job as the gatekeepers of their work. Consciously build a positive value system and promote the core values of socialism. Also, adequately target the characteristics of teenage audiences and maintain the communication effect to achieve considerable strength. Spreading influence makes teenagers form a certain degree of psychological trust in artistic aesthetics. The impact of movies on teenagers has both advantages and disadvantages. The importance of the mass media is not direct and immediate, but indirect, long-term and subtle, and varies from person to person. The judgment of the impact of movies on teenagers requires rational thinking. Also, try to make good use of its long-term skills to form positive energy to promote and guide the teenagers of Pakistan, and strive to put an end to all film and television works that are not conducive to the growth of children spread. 


\section{REFERENCES}

Ali, A. Z., \& Ali, N. H. (2015). Teen dating Violence. International Journal of Women Empowerment. https://doi.org/10.29052/2413-4252.v1.i1.2015.30-32

Anderson, C. a, \& Warburton, W. a. (2012). The impact of violent video games : An overview. Growing Up Fast and Furious.

Barlińska, J., Szuster, A., \& Winiewski, M. (2013). Cyberbullying among adolescent bystanders: Role of the communication medium, form of violence, and empathy. Journal of Community and Applied Social Psychology. https:// doi.org/10.1002/casp. 2137

Cardwell, M. S. (2013). Video media-induced aggressiveness in children. In Southern Medical Journal. https://doi.org/10.1097/SMJ.0b013e3182a5eef4

Dai, Q., Zhao, R. W., Wu, Z., Wang, X., Gu, Z., Wu, W., \& Jiang, Y. G. (2015). Fudan-Huawei at MediaEval 2015: Detecting violent scenes and affective impact in movies with deep learning. CEUR Workshop Proceedings.

Huesmann, L. R. (2007). The Impact of Electronic Media Violence: Scientific Theory and Research. In Journal of Adolescent Health. https://doi.org/10.1016/j. jadohealth.2007.09.005

Jang, J. Y., Han, K., Shih, P. C., \& Lee, D. (2015). Generation like: Comparative characteristics in instagram. Conference on Human Factors in Computing Systems - Proceedings. https://doi.org/10.1145/2702123.2702555

Matrix, S. (2014). The Netflix Effect: Teens, Binge Watching, and On-Demand Digital Media Trends. Jeunesse: Young People, Texts, Cultures. https://doi. org/10.1353/jeu.2014.0002

Pavlova, M. K., \& Silbereisen, R. K. (2015). Cyberbullying among Adolescent Bystanders: Role of the Communication Medium, Form of Violence, and Empathy. Journal of Community \& Applied Social Psychology. https://doi. org/10.1002/casp

Wang, K., Zhang, Z., \& Wang, L. (2012). Violence video detection by discriminative slow feature analysis. Communications in Computer and Information Science. https://doi.org/10.1007/978-3-642-33506-8 18

Wartella, E., Rideout, V., Montague, H., Beaudoin-Ryan, L., \& Lauricella, A. (2016). Teens, health and technology: A national survey. Media and Communication. https://doi.org/10.17645/mac.v4i3.515 